\title{
Phase diagram of neutral quark matter in nonlocal chiral quark models
}

\author{
D. Gómez Dumm, ${ }^{1,2, *}$ D. B. Blaschke, ${ }^{3, \dagger}$ A. G. Grunfeld, ${ }^{4, \$}$ and N. N. Scoccola ${ }^{2,4,5,8}$ \\ ${ }^{1}$ IFLP, CONICET Dpto. de Física, Universidad Nacional de La Plata, \\ C.C. 67, 1900 La Plata, ArgentinaCONICET, Rivadavia 1917, 1033 Buenos Aires, Argentina \\ ${ }^{2}$ Gesellschaft für Schwerionenforschung (GSI), Planckstr. 1, 64291 Darmstadt, Germany \\ ${ }^{3}$ Bogoliubov Laboratory of Theoretical Physics, JINR Dubna, Joliot-Curie Street 6, 141980 Dubna, Russia \\ ${ }^{4}$ Physics Department, Comisión Nacional de Energía Atómica, Av. Libertador 8250, 1429 Buenos Aires, Argentina \\ ${ }^{5}$ Universidad Favaloro, Solís 453, 1078 Buenos Aires, Argentina
}

(Received 28 December 2005; published 20 June 2006)

\begin{abstract}
We consider the phase diagram of two-flavor quark matter under neutron star constraints for two nonlocal, covariant quark models within the mean-field approximation. In the first case (Model I) the nonlocality arises from the regularization procedure, motivated by the instanton liquid model, whereas in the second one (Model II) a separable approximation of the one-gluon exchange interaction is applied. We find that Model II predicts a larger quark mass gap and a chiral symmetry breaking (CSB) phase transition line which extends $15-20 \%$ further into the phase diagram spanned by temperature $(T)$ and chemical potential $(\mu)$. The corresponding critical temperature at $\mu=0, T_{c}(0) \simeq 140 \mathrm{MeV}$, is in better accordance to recent lattice QCD results than the prediction of the standard local NJL model, which exceeds $200 \mathrm{MeV}$. For both Model I and Model II we have considered various coupling strengths in the scalar diquark channel, showing that different low-temperature quark matter phases can occur at intermediate densities: a normal quark matter (NQM) phase, a two-flavor superconducting (2SC) quark matter phase and a mixed 2SC-NQM phase. Although in most cases there is also a gapless 2SC phase, this occurs in general in a small region at nonzero temperatures, thus its effect should be negligible for compact star applications.
\end{abstract}

DOI: 10.1103/PhysRevD.73.114019

\section{INTRODUCTION}

Within the last decade the investigation of the thermodynamics and phase structure of strongly interacting matter has been driven by the results of the experimental programs with ultrarelativistic heavy ion beams at CERN-SPS and BNL-RHIC [1] as well as by the unprecedented quality of data from lattice QCD simulations [2]. A new picture of the state of matter created in these experiments has emerged, according to which the physical nature of the sought-for quark-gluon plasma (QGP) is a perfect liquid of strongly correlated hadronlike resonances rather than an ideal gas of quasifree quarks and gluons [3,4]. The persistence of a strong, nonperturbative coupling in the QGP can be supposed as a prerequisite of a successful modelling of the QCD phase diagram. Turning to the domain of finite chemical potentials, guidance from lattice QCD is limited to $\mu \leq T$ (where Taylor expansion techniques can be applied), and experimental programmes such as CBM at FAIR Darmstadt are yet in the stage of preparation with a planned begin of operation in 2014. Therefore, predictions for the QCD thermodynamics at low temperatures and high baryon densities $\mu \geq T$, where the critical point and the regions of color superconducting quark matter are expected in the QCD phase diagram [5], have to be developed

\footnotetext{
*Electronic address: dumm@fisica.unlp.edu.ar

†Electronic address: blaschke@theory.gsi.de;

Present address: Institute of Physics, University of Rostock, D-18055 Rostock, Germany

Electronic address: grunfeld@tandar.cnea.gov.ar

${ }^{\S}$ Electronic address: scoccola@tandar.cnea.gov.ar
}

PACS numbers: 12.38.Mh, 24.85.+p, 26.60.+c, 97.60. $-\mathrm{s}$

within effective models for nonperturbative QCD and to be tested against observational constraints from neutron stars [6]. For a recently developed testing scheme we refer to [7].

After the early discussion on color superconducting dense quark matter [8] was revived for nonperturbatively strong couplings within effective quark models [9], a great variety of possible diquark pairing patterns and corresponding phases has been explored [5] and estimates for the order of magnitude of the pairing gaps have been given. One of the central questions for phenomenological applications in compact stars, where electric and charge neutrality has to be imposed [10,11], concerns the number of active flavors. It turns out that at low temperatures one obtains a sequential melting pattern of the light and strange quark chiral condensates, which is rather insensitive to the details of the four-momentum dependence of the interaction, but crucially dependent on whether also the strange quark mass is determined self-consistently (see [12] for an early work within the covariant chiral quark model). The present 'state-of-the-art' for the description of color superconducting phases is represented by the fully selfconsistent mean-field three-flavor Nambu-Jona-Lasinio (NJL) model calculations of Refs. [13-15]. The results of these studies indicate that under compact star conditions, i.e. color and electric charge neutrality together with $\beta$-equilibrium, the two-flavor color superconducting (2SC) phase is favored over the three-flavor color-flavorlocking (CFL) one. This outdates earlier results along the lines of, e.g., Ref. [11] where the strange quark mass has been set to a constant, small value. Even if the third quark 
flavor occurs at not too high densities to be in principle realized in compact star interiors, the star configurations with CFL quark cores turn out to be hydrodynamically unstable [16]. For a more detailed introduction to the recent status of dense quark matter in the NJL model, see [17].

Studies of neutral 2SC quark matter consider also the presence of a so-called gapless (g2SC) phase [18], which is found to occur at intermediate temperatures and chemical potentials. Going beyond the NJL theory, results arising from a noncovariant nonlocal quark model $[19,20]$ with Gaussian regulator functions show that the 2SC phase is not present in asymmetric quark matter for standard values of the diquark coupling [21]. In this model, however, for strong diquark couplings one does find a $2 \mathrm{SC}$ phase, together with a g2SC and a mixed normal quark matter (NQM)-2SC phase in which the electric charge neutrality is satisfied only globally [22-24].

As the structure of the quark matter phase diagram within NJL-type models is settling, it is important to investigate the situation in the case of effective models that go beyond those used in Refs. [11,13-15,21], in the sense that they include fully covariant nonlocal interactions. Nonlocality arises naturally in the context of several quite successful approaches to low-energy quark dynamics as, for example, the instanton liquid model [25] and the Schwinger-Dyson resummation techniques [26]. The same happens in lattice QCD [27]. It has been also argued that nonlocal models have several advantages over the local ones (e.g., the NJL model [28] and its generalizations). Indeed, nonlocal interactions regularize the model in such a way that anomalies are preserved [29] and charges properly quantized, the effective interaction is finite to all orders in the loop expansion and there is no need to introduce extra cut-offs. Soft regulators such as Gaussian functions lead to small next-to-leading order corrections [30,31], etc. This type of models has been successfully used to investigate meson [32-35] and baryon [36-38] properties at vanishing temperature and chemical potential. The phase diagram of isospin symmetric matter has also been studied within this context [39-43]. The aim of the present work is to extend these analyses to the case in which compact star conditions are imposed. We will consider two-flavor versions of the models, since (as mentioned below) effects arising from the strange quark can be safely neglected in the region of intermediate chemical potentials to be covered. The investigation of the question whether the results of the above referenced studies will remain qualitatively unchanged after the inclusion of correlations beyond the mean-field level goes beyond the scope of the present work and deserves a separate study. It should be noticed, however, that in the nonstrange case considered here the relevant color superconducting condensate transforms as a singlet representation of the global $\mathrm{SU}(2)_{L} \otimes \mathrm{SU}(2)_{R}$ chiral group. Thus, no global symmetry is broken by the $2 \mathrm{SC}$ ground state, which implies that there are no Goldstone bosons that could further condense.

The paper is organized as follows. In Sec. II we introduce the model and derive some useful analytical expressions. In Sec. III we present the numerical results obtained for the case of a Gaussian regulator, considering different ratios between the coupling constants. Then, in Sec. IV we discuss the features of the obtained phase diagrams, and in Sec. V we present our conclusions.

\section{FORMALISM}

Let us begin by stating the Euclidean action for the nonlocal chiral quark model in the case of two light flavors and antitriplet diquark interactions,

$$
\begin{aligned}
S_{E}= & \int d^{4} x\left\{\bar{\psi}(x)(-i \not \supset+m) \psi(x)-\frac{G}{2} j_{M}^{f}(x) j_{M}^{f}(x)\right. \\
& \left.-\frac{H}{2}\left[j_{D}^{a}(x)\right]^{\dagger} j_{D}^{a}(x)\right\} .
\end{aligned}
$$

Here $m$ is the current quark mass, which is assumed to be equal for $u$ and $d$ quarks. The nonlocality can be introduced now in different ways [44]. In what follows we will work within two alternative scenarios, that we call "Model I" and "Model II", in which the mesonic current $j_{M}(x)$ and the diquark current $j_{D}(x)$ in Eq. (1) are given by nonlocal operators. In the case of Model I [33,34], the effective interactions are based on an instanton liquid picture of QCD. The nonlocal currents read

$$
\begin{aligned}
& j_{M}^{f}(x)=\int d^{4} y d^{4} z r(y-x) r(x-z) \bar{\psi}(y) \Gamma_{f} \psi(z), \\
& j_{D}^{a}(x)=\int d^{4} y d^{4} z r(y-x) r(x-z) \bar{\psi}_{C}(y) i \gamma_{5} \tau_{2} \lambda_{a} \psi(z),
\end{aligned}
$$

where we have defined $\psi_{C}(x)=\gamma_{2} \gamma_{4} \bar{\psi}^{T}(x)$ and $\Gamma_{f}=$ $\left(\mathbf{1}, i \gamma_{5} \vec{\tau}\right)$, while $\vec{\tau}$ and $\lambda_{a}$, with $a=2,5,7$ stand for Pauli and Gell-Mann matrices acting on flavor and color spaces, respectively.

On the other hand, Model II [32,45] arises from a separable form of the effective one-gluon exchange (OGE) picture. In this case the nonlocal currents $j_{M, D}(x)$ are given by

$$
\begin{aligned}
& j_{M}^{f}(x)=\int d^{4} z g(z) \bar{\psi}\left(x+\frac{z}{2}\right) \Gamma_{f} \psi\left(x-\frac{z}{2}\right), \\
& j_{D}^{a}(x)=\int d^{4} z g(z) \bar{\psi}_{C}\left(x+\frac{z}{2}\right) i \gamma_{5} \tau_{2} \lambda_{a} \psi\left(x-\frac{z}{2}\right) .
\end{aligned}
$$

The functions $r(x-y)$ and $g(z)$ in Eqs. (2) and (3) are nonlocal regulators characterizing the corresponding interactions. It is convenient to translate them into momentum space. Since Lorentz invariance implies that they can only be functions of $p^{2}$, we will use for the Fourier transforms of these regulators the forms $r\left(p^{2}\right)$ and $g\left(p^{2}\right)$ from now on. 
The current-current interaction in Eq. (1) is quite common to effective theories for low-energy QCD such as the NJL model. The momentum dependence introduced by the functions $r\left(p^{2}\right)$ and $g\left(p^{2}\right)$ is a generalization of that model, and can be chosen in such a way that the dynamical mass function of the quark propagator reproduces some features obtained in lattice QCD analyses. It could entail, e.g., the possibility of complex conjugate mass poles of the quark propagator, in agreement with results arising from the Schwinger-Dyson equation approach to QCD. The index structure of the vertices $\Gamma_{f}$ and also the ratio of the coupling constants $H / G$ can be obtained from a Fierz rearrangement of e.g. the OGE interaction, as detailed in [17] (see also references therein). For the OGE, as well as for the instanton liquid model, one obtains $H / G=0.75$. However, since a precise derivation of the effective couplings from QCD is not known, there is a significant theoretical uncertainty in this value. In fact, so far there is no strong phenomenological constraint on $H / G$, except for the fact that values larger that $H / G \sim 1$ are quite unlikely to be realized in QCD, since they might lead to color symmetry breaking in the vacuum. We will leave this ratio as a free parameter, analyzing the results obtained for values lying within a range from 0.5 to 1 .

The partition function of the system at temperature $T$ and quark chemical potentials $\mu_{f c}$ is given by

$$
Z=\int \mathcal{D} \bar{\psi} \mathcal{D} \psi e^{-S_{E}\left(\mu_{f c}, T\right)},
$$

where the Euclidean action is obtained from Eq. (1) by going to momentum space and performing the replacements

$$
p_{4} \rightarrow \omega_{n}-i \mu_{f c}, \quad \int \frac{d^{4} p}{(2 \pi)^{4}} \rightarrow T \sum_{n=-\infty}^{\infty} \int \frac{d^{3} \vec{p}}{(2 \pi)^{3}} .
$$

Here $p_{4}$ is the fourth component of the (Euclidean) momentum of a quark carrying flavor $f$ and color $c$, and $\omega_{n}$ are the Matsubara frequencies corresponding to fermionic modes, $\omega_{n}=(2 n+1) \pi T$. We are assuming here that quark interactions depend on the temperature and chemical potentials only through the arguments of the regulators. Note that, as required for the applications we are interested in, we have introduced in Eq. (4) different chemical potentials for each quark flavor and color.

To proceed it is convenient to perform a standard bosonization of the theory. This procedure is described, e.g., in Refs. [44,45]. Thus, we introduce the bosonic fields $\sigma$, $\pi_{a}$ and $\Delta_{a}$, and integrate out the quark fields. In what follows, we work within the mean-field approximation (MFA), in which these bosonic fields are replaced by their vacuum expectation values $\bar{\pi}_{a}=0, \bar{\sigma}$ and $\bar{\Delta}_{a}$. Moreover, we adopt the usual 2SC ansatz $\bar{\Delta}_{5}=\bar{\Delta}_{7}=0, \bar{\Delta}_{2}=\bar{\Delta}$. We have assumed other possible condensates to be negligible, following previous analyses carried out in the NJL model framework [5,17]. Within the MFA, and employing the Nambu-Gorkov formalism, the thermodynamical potential per unit volume can be written as

$$
\begin{aligned}
\Omega^{\mathrm{MFA}}= & -\frac{T}{V} \ln Z^{\mathrm{MFA}} \\
= & \frac{\bar{\sigma}^{2}}{2 G}+\frac{|\bar{\Delta}|^{2}}{2 H}-\frac{T}{2} \sum_{n=-\infty}^{\infty} \int \frac{d^{3} \vec{p}}{(2 \pi)^{3}} \\
& \times \operatorname{lndet}\left[\frac{1}{T} S^{-1}(\bar{\sigma}, \bar{\Delta})\right] .
\end{aligned}
$$

Here the inverse propagator $S^{-1}(\bar{\sigma}, \bar{\Delta})$ is a $48 \times 48$ matrix in Dirac, flavor, color and Nambu-Gorkov spaces, given by

$$
\left.\begin{array}{cccc}
0 & 0 & \gamma_{5} \tau_{2}^{c} \Delta & 0 \\
0 & 0 & 0 & 0 \\
-\gamma_{5} \tau_{2}^{c} \Delta^{*} & 0 & 0 & 0 \\
0 & 0 & 0 & 0 \\
-\not p_{u r}^{-}+\Sigma_{u r}{ }^{*} & 0 & 0 & 0 \\
0 & -\not p_{u b}^{-}+\Sigma_{u b}^{*} & 0 & 0 \\
0 & 0 & -\not p_{d r}^{-}+\Sigma_{d r}^{*} & 0 \\
0 & 0 & 0 & -\not p_{d b}^{-}+\Sigma_{d b}{ }^{*}
\end{array}\right)
$$

where we have used the definitions

$$
\begin{gathered}
p_{f c}^{ \pm}=\left(\omega_{n} \mp i \mu_{f c}, \vec{p}\right), \\
\Sigma_{f c}=m+\bar{\sigma} h\left(p_{f c}^{+}, p_{f c}^{+}\right), \\
\Delta=\bar{\Delta} h\left(p_{u r}^{+}, p_{d r}^{-}\right),
\end{gathered}
$$

with $f=u, d$ and $c=r, g, b$. The functions $h(p, q)$ have been introduced in order to have a common notation for both Model I and Model II. One has

$$
h(s, t)= \begin{cases}r\left(s^{2}\right) r\left(t^{2}\right) & (\text { Model I }) \\ g\left(\left[\frac{s+t}{2}\right]^{2}\right) & (\text { Model II })\end{cases}
$$

We have taken into account that, as we will see below, the 
usual 2SC ansatz implies $\mu_{f r}=\mu_{f g}$. In Eq. (7), entries with subindices $u r$ and $d r$ are intended to be multiplied by an $\mathbb{1}_{2 \times 2}$ matrix in $r g$ space, while $\tau_{2}^{c}$ stands for a $\tau_{2}$ Pauli matrix acting in this space.

The determinant of $S^{-1}$ can be analytically evaluated, leading to

$$
\Omega^{\mathrm{MFA}}=\frac{\bar{\sigma}^{2}}{2 G}+\frac{\bar{\Delta}^{2}}{2 H}-T \sum_{n=-\infty}^{\infty} \int \frac{d^{3} \vec{p}}{(2 \pi)^{3}} \sum_{c=r, g, b} \ln \frac{\left|A_{c}\right|^{2}}{T^{8}},
$$

where

$$
\begin{aligned}
A_{c}= & \left(p_{u c}^{+2}+\Sigma_{u c}^{2}\right)\left(p_{d c}^{-2}+\Sigma_{d c}^{* 2}\right)+\left(1-\delta_{b c}\right) \Delta^{2} \\
& \times\left[\Delta^{2}+2\left(p_{u c}^{+} \cdot p_{d c}^{-}\right)+2 \Sigma_{u c} \Sigma_{d c}^{*}\right] .
\end{aligned}
$$

For finite values of the current quark mass, $\Omega^{\mathrm{MFA}}$ turns out to be divergent. The regularization procedure used here amounts to define

$$
\Omega_{(\text {reg })}^{\mathrm{MFA}}=\Omega^{\mathrm{MFA}}-\Omega^{\mathrm{free}}+\Omega_{(\mathrm{reg})}^{\mathrm{free}},
$$

where $\Omega^{\text {free }}$ is obtained from Eq. (12) by setting $\bar{\Delta}=\bar{\sigma}=$ 0 , and $\Omega_{(\mathrm{reg})}^{\mathrm{free}}$ is the regularized expression for the thermodynamical potential of a free fermion gas,

$$
\begin{aligned}
\Omega_{(\text {reg })}^{\text {free }} & =-2 T \int \frac{d^{3} \vec{p}}{(2 \pi)^{3}} \sum_{f, c}\{\ln [1 \\
& \left.\left.=e^{-\left(\sqrt{\vec{p}+m^{2}}-\mu_{f c}\right) / T}\right]+\ln \left[1+e^{-\left(\sqrt{\vec{p}+m^{2}}-\mu_{f c}\right) / T}\right]\right\} .
\end{aligned}
$$

The mean-field values $\bar{\sigma}$ and $\bar{\Delta}$ are obtained from the coupled gap equations

$$
\begin{gathered}
\frac{d \Omega_{(\mathrm{reg})}^{\mathrm{MFA}}}{d \bar{\Delta}}=\bar{\Delta}\left(1-16 H T D_{u d}\right)=0, \\
\frac{d \Omega_{(\mathrm{reg})}^{\mathrm{MFA}}}{d \bar{\sigma}}=\bar{\sigma}-4 G T\left(S_{u d}+S_{d u}\right)=0,
\end{gathered}
$$

where we have defined

$$
\begin{aligned}
D_{i j}= & D_{j i} \\
= & \operatorname{Re} \sum_{n=-\infty}^{\infty} \int \frac{d^{3} \vec{p}}{(2 \pi)^{3}} h^{2}\left(p_{u r}^{+}, p_{d r}^{-}\right) \\
& \times \frac{\Delta^{2}+\left(p_{i r}^{+} \cdot p_{j r}^{-}\right)+\Sigma_{i r} \Sigma_{j r}^{*}}{A_{r}},
\end{aligned}
$$

$$
\begin{aligned}
S_{i j}= & \operatorname{Re} \sum_{n=-\infty}^{\infty} \int \frac{d^{3} \vec{p}}{(2 \pi)^{3}}\left\{2 h\left(p_{i r}^{+}, p_{i r}^{+}\right)\right. \\
& \times \frac{\sum_{i r}\left(p_{j r}^{-2}+\Sigma_{j r}^{* 2}\right)+\Delta^{2} \Sigma_{j r}^{*}}{A_{r}} \\
& \left.+h\left(p_{i b}^{+}, p_{i b}^{+}\right) \frac{\Sigma_{i b}}{p_{i b}^{+2}+\Sigma_{i b}^{2}}\right\} .
\end{aligned}
$$

So far we have introduced different chemical potentials for each quark flavor and color. However, not all of them are in general independent quantities. For the description of quark matter in the core of neutron stars, we require the system to be color and electric charge neutral (for a further discussion on the issue of color neutrality and color singletness we refer to Refs. [11,46]). Thus, within the previously introduced 2SC ansatz, only one color-dependent chemical potential is needed [47], and the $\mu_{f c}$ can be written in terms of only three independent quantities: the baryonic chemical potential $\mu_{B}$, the quark electric chemical potential $\mu_{Q_{q}}$ and the color chemical potential $\mu_{8}$. Defining $\mu \equiv$ $\mu_{B} / 3$, the corresponding relations read

$$
\begin{aligned}
& \mu_{u r}=\mu_{u g}=\mu+\frac{2}{3} \mu_{Q_{q}}+\frac{1}{3} \mu_{8} \\
& \mu_{d r}=\mu_{d g}=\mu-\frac{1}{3} \mu_{Q_{q}}+\frac{1}{3} \mu_{8} \\
& \mu_{u b}=\mu+\frac{2}{3} \mu_{Q_{q}}-\frac{2}{3} \mu_{8} \\
& \mu_{d b}=\mu-\frac{1}{3} \mu_{Q_{q}}-\frac{2}{3} \mu_{8}
\end{aligned}
$$

Now, in the core of neutron stars, in addition to quark matter we have electrons. Thus, within the mean-field approximation for the quark matter, and considering the electrons as a free Dirac gas, the full grand canonical potential is given by

$$
\Omega^{\mathrm{full}}=\Omega_{(\mathrm{reg})}^{\mathrm{MFA}}+\Omega^{e},
$$

where

$$
\Omega^{e}=-\frac{1}{12 \pi^{2}}\left(\mu_{e}^{4}+2 \pi^{2} T^{2} \mu_{e}^{2}+\frac{7 \pi^{4}}{15} T^{4}\right),
$$

$\mu_{e}$ being the electron chemical potential. For simplicity we have neglected here the electron mass.

In addition, it is necessary to take into account that quark matter has to be in $\beta$-equilibrium with electrons through the beta-decay reaction

$$
d \rightarrow u+e+\bar{\nu}_{e} .
$$

Thus, assuming that antineutrinos escape from the stellar core, we must have

$$
\mu_{d c}-\mu_{u c}=-\mu_{Q_{q}}=\mu_{e} .
$$


If we impose the requirements of electric and color charge neutrality, $\mu_{e}$ and $\mu_{8}$ become fixed by the conditions of vanishing electric and color densities:

$$
\begin{aligned}
\rho_{Q_{\mathrm{tot}}} & =\rho_{Q_{q}}-\rho_{e}=\sum_{c=r, g, b}\left(\frac{2}{3} \rho_{u c}-\frac{1}{3} \rho_{d c}\right)-\rho_{e}=0, \\
\rho_{8} & =\frac{1}{\sqrt{3}} \sum_{f=u, d}\left(\rho_{f r}+\rho_{f g}-2 \rho_{f b}\right)=0,
\end{aligned}
$$

where

$$
\begin{aligned}
& \rho_{e}=-\frac{\partial \Omega}{\partial \mu_{e}}=-\frac{\partial \Omega^{e}}{\partial \mu_{e}}, \\
& \rho_{f c}=-\frac{\partial \Omega}{\partial \mu_{f c}}=-\frac{\partial \Omega_{(\mathrm{reg})}^{\mathrm{MFA}}}{\partial \mu_{f c}} .
\end{aligned}
$$

Consequently, in the physical situation we are interested in, for each value of $T$ and $\mu$ we should find the values of $\bar{\Delta}$, $\bar{\sigma}, \mu_{e}$ and $\mu_{8}$ that solve Eqs. (16) and (17), supplemented by Eqs. (24) and (25).

The electron density can be evaluated analytically, leading to

$$
\rho_{e}=\frac{\mu_{e}}{3 \pi^{2}}\left(\mu_{e}^{2}+\pi^{2} T^{2}\right) .
$$

On the other hand, from Eqs. (12), (14), and (15), the quark densities can be expressed as

$$
\rho_{(\mathrm{reg}) f c}^{\mathrm{MFA}}=\rho_{f c}^{\mathrm{MFA}}-\rho_{f c}^{\mathrm{free}}+\rho_{(\mathrm{reg}) f c}^{\mathrm{free}} .
$$

Then a straightforward calculation leads to

$$
\rho_{f c}^{\mathrm{MFA}}=2 T \sum_{n=-\infty}^{\infty} \int \frac{d^{3} \vec{p}}{(2 \pi)^{3}} \operatorname{Re}\left(\frac{1}{A_{c}} \frac{\partial A_{c}}{\partial \mu_{f c}}\right),
$$

with

$$
\begin{aligned}
\frac{\partial A_{c}}{\partial \mu_{u c}}= & -2\left(i \omega_{n}+\mu_{u c}\right)\left(p_{d c}^{-2}+\Sigma_{d c}^{* 2}\right)\left(1+2 \Sigma_{u c} \Sigma_{u c}^{\prime}\right) \\
& -2\left(1-\delta_{b c}\right) \Delta^{2}\left[2 \Sigma_{d c}^{*} \Sigma_{u c}^{\prime}\left(i \omega_{n}+\mu_{u c}\right)\right. \\
& \left.+i \omega_{n}-\mu_{d c}\right]-4 i\left(1-\delta_{b c}\right) \bar{\Delta} \Delta\left[\Delta^{2}+\left(p_{u c}^{+} \cdot p_{d c}^{-}\right)\right. \\
& \left.+\Sigma_{u c} \Sigma_{d c}^{*}\right]\left.\frac{\partial h\left(t, p_{d c}^{-}\right)}{\partial t_{4}}\right|_{t=p_{u c}^{+}}
\end{aligned}
$$

where we have defined $\Sigma_{f c}^{\prime}=\bar{\sigma} \partial h(t, t) /\left.\partial t^{2}\right|_{t^{2}=p_{f c}^{+2}}$.

The corresponding expressions for $\rho_{d c}$ are obtained by simply exchanging $u$ and $d$ and taking the complex conjugate, while the expressions for $\rho_{f c}^{\text {free }}$ can be easily obtained from $\rho_{f c}^{\mathrm{MFA}}$ by setting $\bar{\sigma}=\bar{\Delta}=0$. Finally, $\rho_{(\text {reg }) f c}^{\mathrm{free}}$ is given by

$$
\begin{aligned}
\rho_{(\text {reg }) f c}^{\mathrm{free}}= & 2 \int \frac{d^{3} \vec{p}}{(2 \pi)^{3}} \int\left[1+\exp \left(\frac{\sqrt{\vec{p}^{2}+m^{2}}-\mu_{f c}}{T}\right)\right]^{-1} \\
& \left.-\left[1+\exp \left(\frac{\sqrt{\vec{p}^{2}+m^{2}}+\mu_{f c}}{T}\right)\right]^{-1}\right\} .
\end{aligned}
$$

\section{NUMERICAL RESULTS}

In this section we present our numerical results, showing the features of the phase diagram and the behavior of relevant physical quantities for Models I and II. According to previous analyses carried out within nonlocal scenarios [42], the results are not expected to show a strong qualitative dependence on the shape of the regulator. Thus we will concentrate here on simple and well-behaved Gaussian regulator functions, taking (in momentum space)

$$
\begin{aligned}
& r\left(p^{2}\right)=\exp \left(-p^{2} / 2 \Lambda^{2}\right) \quad \text { (Model I) } \\
& g\left(p^{2}\right)=\exp \left(-p^{2} / \Lambda^{2}\right) \quad(\text { Model II })
\end{aligned}
$$

Here $\Lambda$ is a free model parameter, playing the rôle of an ultraviolet cut-off. We have chosen a different normalization for Models I and II in view of the relation between the respective regulating functions [see Eq. (11)], which determine the low $T$ and $\mu$ phenomenology.

\section{A. Parametrization}

For definiteness, for both Models I and II we choose here input parameters $m, \Lambda$ and $G$ which allow to reproduce the empirical values for the pion mass $m_{\pi}=139 \mathrm{MeV}$ and decay constant $f_{\pi}=92.4 \mathrm{MeV}$, and lead to a phenomenologically acceptable value for the chiral condensates at vanishing $T$ and $\mu_{f c}$. For Gaussian regulators, taking into account the chosen normalization of the cut-offs, it is seen that within the MFA both models lead to the same expressions for the considered physical quantities at $T=\mu=0$. However, this is not the case when one goes beyond the MFA [48]. In particular, the expressions for the pion mass and decay constant are different (they are still coincident only in the chiral limit), and it is necessary to use different sets of input parameters. The parameters considered here for Model I are $m=5.14 \mathrm{MeV}, \Lambda=971 \mathrm{MeV}$ and $G \Lambda^{2}=15.41$, while for Model II we have taken $m=$ $5.12 \mathrm{MeV}, \Lambda=827 \mathrm{MeV}$ and $G \Lambda^{2}=18.78$. With these sets we get for both models a phenomenologically reasonable value for the chiral condensate, namely $\langle 0|\bar{q} q| 0\rangle^{1 / 3}=$ $-250 \mathrm{MeV}$. The remaining free parameter is the coupling strength $H$ in the scalar diquark channel. In order to fix its value by hadron phenomenology at zero $T$ and $\mu$, one would have to solve the Faddeev-type equations for baryons as three-quark bound states which result from the quantization of chiral quark models of the type considered in the present paper after bosonization in meson and diquark channels (see [49] and Refs. therein; for more elabo- 
rate recent calculations of nucleon properties see [37,50]). Although in principle this is possible, we refrain from fixing $H$ by hadron phenomenology within the present exploratory study of the quark matter phase diagram and rather choose different values for the coupling ratio $H / G$ in the range from 0.5 to 1 .

\section{B. Order parameters and phase transitions}

For fixed values of the temperature $T$ and the chemical potential $\mu\left(\mu=\mu_{B} / 3\right)$, the mean-field values $\bar{\sigma}$ and $\bar{\Delta}$, as well as the chemical potentials $\mu_{e}$ and $\mu_{8}$, can be numeri- cally obtained from the gap Eqs. (16) and (17), together with the conditions of $\beta$-equilibrium and vanishing color and electric charge densities, Eqs. (24) and (25). Let us begin by considering the case $H / G=0.75$, which is motivated by various effective models of quark-quark interactions. Our results for $\bar{\sigma}, \bar{\Delta}, \mu_{e}$ and $\mu_{8}$ are shown in Fig. 1, where we plot these quantities as functions of $\mu$ for different representative values of the temperature. Left and right panels correspond to Models I and II, respectively.

For $T=0$ (solid lines), at low chemical potentials the system is for both Models I and II in a chiral symmetry broken phase (CSB), where quarks acquire large dynami-
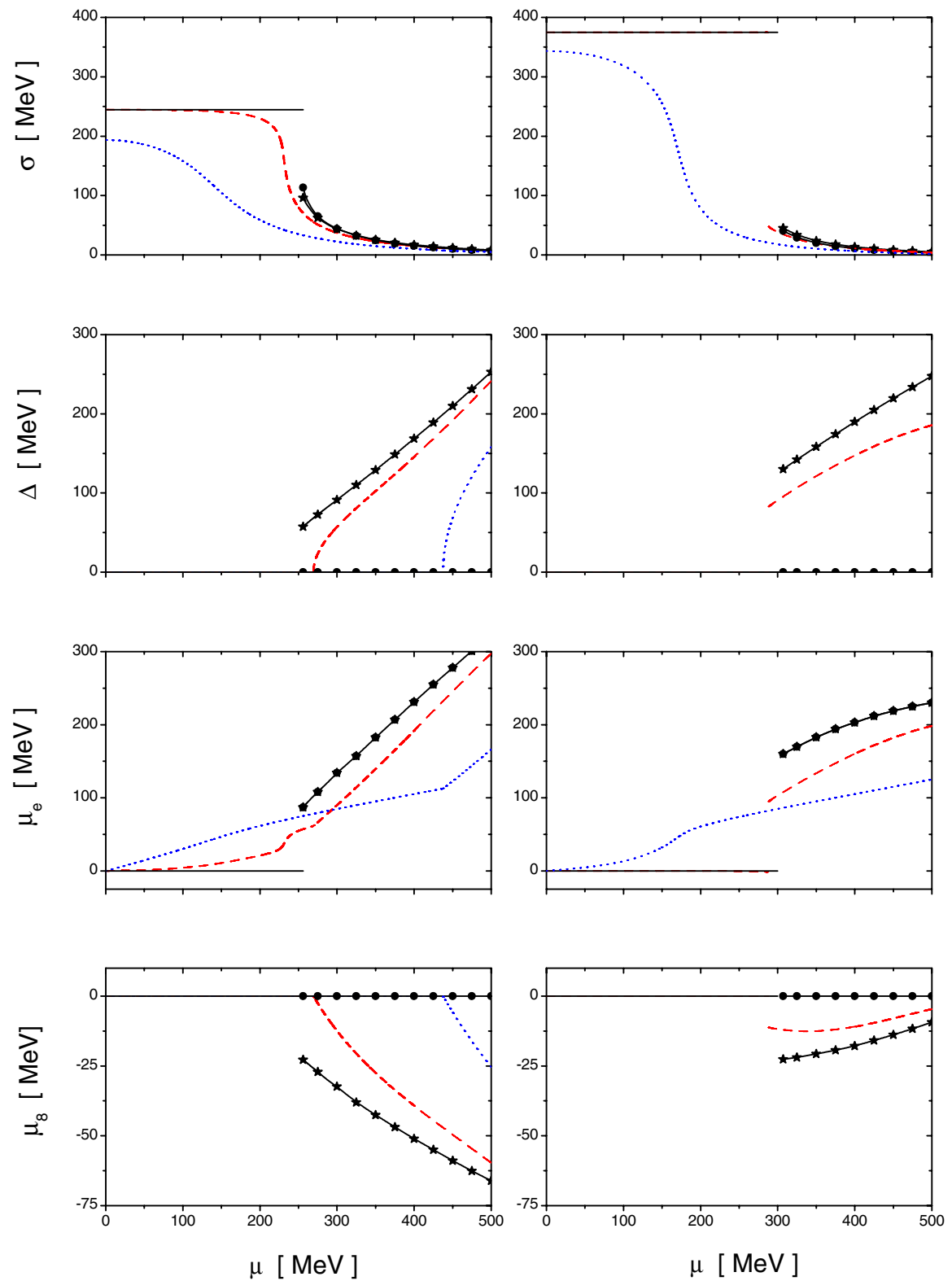

FIG. 1 (color online). Behavior of the mean fields $\bar{\sigma}$ and $\bar{\Delta}$ and the chemical potentials $\mu_{e}$ and $\mu_{8}$ for Models I (left) and II (right) as a function of the chemical potential, for three different values of the temperature. The curves correspond to the case $H / G=3 / 4$. Full lines correspond to $T=0$, dashed lines to $T=40 \mathrm{MeV}$ and dotted lines to $T=100 \mathrm{MeV}$. In the case of $T=0$, lines marked with stars and dots correspond to the 2SC and NQM phases, respectively. 
cal masses. By increasing the chemical potential one reaches a first order phase transition, in which the chiral symmetry is approximately restored, and a certain volume fraction of the quark matter undergoes a transition to the 2SC phase coexisting with the remaining normal quark matter (NQM) phase. The chemical potential $\mu_{e}$ (which for $T=0$ vanishes in the CSB region) also shows a discontinuity across the transition. The new 2SC-NQM mixed phase is a way in which the system realizes the constraint of electric neutrality globally: the coexisting phases have opposite electric charges which neutralize each other, at a common equilibrium pressure. In its simplest realization, this mixed phase is treated within an approximation in which Coulomb and surface energies are neglected (see Ref. [51]). For color superconducting quark matter this realization of charge neutrality has been considered e.g. in Ref. [24] for the NJL model and in Ref. [21] for the instantaneous nonlocal quark model. The discussion of inhomogeneous mixed phases, which are not yet fully understood, crucially depends on the assumptions for the surface tension and charge screening effects (see e.g. Ref. [52]) and goes beyond the scope of the present investigation. On the other hand, following Refs. [23,53], we have imposed color neutrality as a local constraint. This is based on the fact that the color Debye screening length is expected to be short and comparable to the interparticle distance in the regime of interest. As a consequence, $\mu_{8}$ turns out to be different in the two components of the mixed phase. However, it should be kept in mind that this chemical potential is in fact an effective quantity that has to be introduced in these kind of models in order to account for the effect of the gauge fields. Namely, as argued in Refs. [54-56], superconducting quark matter is expected to be automatically color neutral in QCD. As expected, the growth of the color chemical potential $\mu_{8}$ in the 2SC component of the mixed phase is approximately proportional to that of the corresponding $\bar{\Delta}$, which governs the amount of breakdown of the color symmetry due to quark pairing.

When the temperature is increased (see dashed curves in Fig. 1, corresponding to $T=40 \mathrm{MeV}$ ), the mixed phase is no longer favored and the system goes into a pure 2SC phase. For $T=40 \mathrm{MeV}$, this shows up as a second order transition in the case of Model I, and a first order transition in the case of Model II. Now, for both models, when one moves along the first order transition line from $T=0$ towards higher temperatures, one arrives at a triple point (3P). At this point the CSB and 2SC phases coexist with a third NQM phase, in which the chiral symmetry is approximately restored and there is no color superconductivity. Finally, if $T$ is still increased, one reaches an "end point" (EP) where the first order transition from CSB to NQM phases becomes a smooth crossover. The behavior of the dynamical masses and the electric chemical potential $\mu_{e}$ along this smooth transition is shown in Fig. 1, see curves corresponding to $T=40 \mathrm{MeV}$ (Model I) and $T=$ $100 \mathrm{MeV}$ (dotted lines, Models I and II).
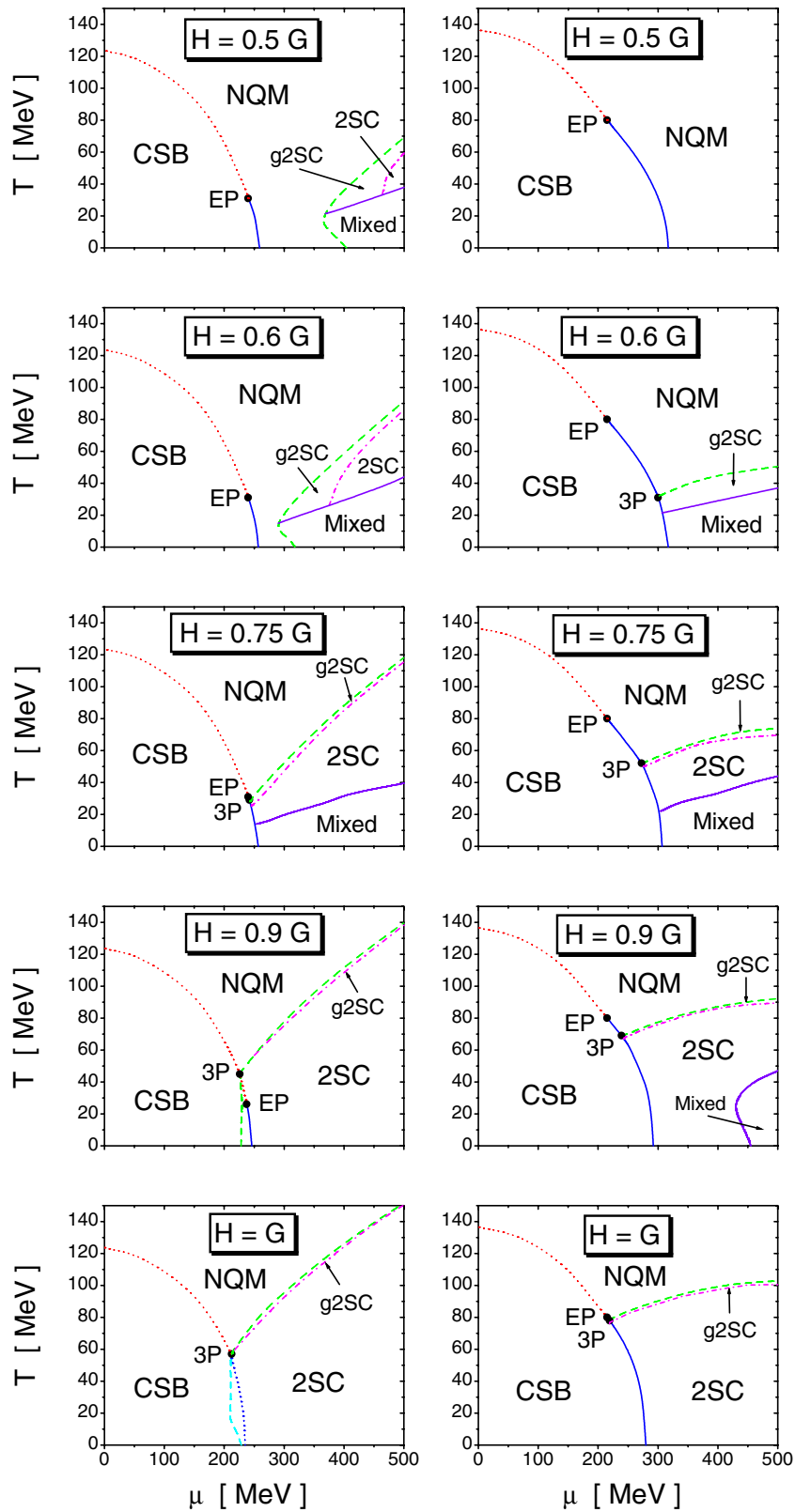

FIG. 2 (color online). Phase diagrams for Models I (left) and II (right) for different values of $H / G$. Full and dashed lines indicate first and second order phase transition curves, respectively, dotted lines correspond to crossoverlike transitions, and dashed lines delimit the gapless 2SC band. Different phases are denoted as NQM (normal quark matter phase), CSB (chiral symmetry broken phase) and 2SC (two-flavor superconducting phase), while the regions marked as "Mixed" correspond to the NQM-2SC mixed phase. Note that in Model I, for $H / G=1$ there is a narrow region in which one has $2 \mathrm{SC}$ while the system is still in a chiral symmetry broken phase. EP and 3P denote the end points and triple points, respectively. 


\section{Quark matter phase diagrams}

The described features of the phase diagrams for Models I and II can be visualized in the graphs shown in Fig. 2, where we plot the transition curves on $T-\mu$ diagrams for different ratios $H / G$, and show the regions corresponding to the different phases and the position of triple and end points. In the graphs, solid and dotted lines correspond to the mentioned first order and crossover transitions, respectively, (in the case of the crossover, the transition point can be defined by considering the maximum of the chiral susceptibility [41]). Between NQM and $2 \mathrm{SC}$ regions we find that in all cases there is a second order phase transition, which corresponds to the dashed lines in the diagrams of Fig. 2. Close to this phase border, the dashed-dotted lines in the graphs delimit a band that corresponds to the so-called gapless $2 \mathrm{SC}$ (g2SC) phase. Here, in addition to the two gapless modes corresponding to the unpaired blue quarks, the presence of flavor asymmetric chemical potentials $\mu_{d c}-\mu_{u c} \neq 0$ gives rise to another two gapless fermionic quasiparticles [18]. Although the corresponding relations cannot be derived analytically owing to the nonlocality of the interactions, the border of the g2SC region can be numerically found. This is done by determining whether for some value of $|\vec{p}|$ the imaginary part of some of the poles of the Euclidean quark propagator vanish in the complex $p_{4}$ plane. From the graphs it is seen that this g2SC band may become relatively significant for low values of $H / G$. However, it never extends up to zero temperatures, therefore this should not represent a robust feature for compact star applications.

\section{DISCUSSION}

Let us discuss some qualitative features of the curves displayed in Figs. 1 and 2. On one hand, for both models the $2 \mathrm{SC}$ phase region becomes larger when the ratio $H / G$ is increased. This is not surprising, since $H$ is the effective coupling governing the quark-quark interaction that gives rise to the pairing. In any case, as a general conclusion it can be stated that, provided the ratio $H / G$ is not too low, these nonlocal schemes favor the existence of color superconducting phases at low temperatures and moderate
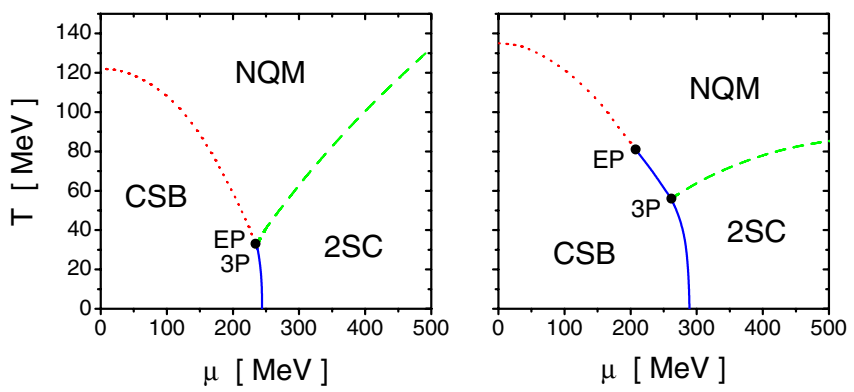

FIG. 3 (color online). Phase diagrams for symmetric matter corresponding to Models I (left) and II (right). Here we have taken $H / G=0.75$. chemical potentials. Indeed, for the parameters considered here, there is no 2SC phase only in the case of Model II, $H / G=0.5$. This is in contrast with the situation in e.g. the NJL model $[13,17]$, where the existence of such a phase turns out to be rather dependent on the input parameters. In addition, our results are qualitatively different from those obtained in the case of the noncovariant nonlocal models [21], where above the chiral phase transition the NQM phase is preferable for values of the coupling ratio $H / G \lesssim$ 0.75 . In those models, a color superconducting quark matter phase can be found only for $H / G \approx 1$.

It is also interesting to compare our results with those obtained for isospin symmetric quark matter. For the same parameter sets, the corresponding phase diagrams for $H / G=0.75$ are shown in Fig. 3. By comparing them with those of Fig. 2, it can be seen that the 2SC region becomes reduced when one imposes color and electric charge neutrality conditions. This is indeed what one would expect, since the condition of electric charge neutrality leads in general to unequal $u$ and $d$ quark densities, disfavoring the $u$ - $d$ pairing. We notice, however, that the effect is relatively small, and the positions of triple and end points as well as the shape of the critical lines remain approximately unchanged. Concerning the shape of the chiral phase transition line $T_{\mathrm{CSB}}(\mu)$, one observes at intermediate temperatures $50 \mathrm{MeV} \lesssim T_{\mathrm{CSB}} \lesssim 100 \mathrm{MeV}$, which are relevant for the future CBM experiment, an approximately linear behavior. This can be seen as an interpolation between a convex shape obtained in NJL or bag models and a concave shape for confining DysonSchwinger equation models, see e.g. Ref. [57]. It is remarkable that thus in the nonlocal covariant models presented here a similarity with confining quark models occurs and that the chiral/deconfinement transition line in the phase diagram resembles very closely the positions of freeze-out parameters in heavy ion collisions.

Finally, we have studied the dependence of the phase transitions on the model parameters, changing the input value of the chiral condensate within a phenomenologically reasonable range $220 \mathrm{MeV} \leq-\langle 0|\bar{q} q| 0\rangle^{1 / 3} \leq$ $280 \mathrm{MeV}$. From this analysis, it is seen that the qualitative features of the phase diagrams are not significantly modified. In particular, it is seen that one finds in general color superconducting phases at low temperatures and moderate chemical potentials, for intermediate values of the ratio $H / G$. In addition, the values for the critical temperature at $\mu=0$ are quite stable, yielding about $120 \mathrm{MeV}$ for Model I and $140 \mathrm{MeV}$ for Model II. This would favor the description given by Model II, in which the result is closer to the values provided by lattice calculations.

\section{CONCLUSIONS}

We have considered the phase diagram of two-flavor quark matter under neutron star constraints for two nonlocal, covariant quark models within the mean-field ap- 
proximation. In the first one the nonlocality was due to the regularization procedure, motivated by the instanton liquid model (Model I), whereas in the second model a separable approximation of the one-gluon exchange interaction was applied (Model II). Although for the Gaussian regulators considered in this work the vacuum gap equations are identical, both models differ in their fluctuation spectrum and therefore in their parameters (current quark mass $m$, coupling strength $G$, UV cut-off $\Lambda$ ), which have been fixed by using input values for the pion mass, the pion decay constant and the chiral condensate. As result of the numerical evaluation of the corresponding gap equations at finite temperature and chemical potential, we have obtained that Model II predicts a larger quark mass gap and a chiral symmetry breaking phase transition line which extends $15-20 \%$ further into the $T-\mu$ plane when compared to Model I. The prediction for the critical temperature at $\mu=0$ in Model II, $T_{\mathrm{CSB}} \sim 140 \mathrm{MeV}$, is closer to the results of recent lattice QCD simulations than the prediction of both Model I and the well-known local NJL model. Considering different values for the coupling strength in the scalar diquark channel, we have found that under neutron star constraints different lowtemperature quark matter phases can occur at intermediate densities: normal quark matter (NQM), pure superconducting (2SC) quark matter and mixed 2SC-NQM phases. The critical temperature for the $2 \mathrm{SC}$ phase transition is a rising function of $\mu$ in the case of Model I whereas it is rather independent of $\mu$ for Model II, due to the different $\mu$ dependences associated with the scalar diquark gaps. A band of gapless $2 \mathrm{SC}$ (g2SC) appears at the border between 2SC and normal quark matter. At large values of the $H / G$ ratio it is given by a tiny strip in the phase diagram, and grows broader at low diquark couplings. However, the g2SC region does not reach zero temperatures, thus it should not represent a robust feature for compact star applications. Our present investigation has been limited to the mean-field approximation and the neglect of the strange quark flavor. None of these approximations is expected to be crucial for compact star applications, and the detailed study of their impact on our results has been left for further development of the present approach.

\section{ACKNOWLEDGMENTS}

The authors are glad to thank D. Aguilera for useful comments and discussions. This work has been supported in part by CONICET and ANPCyT (Argentina), under grants No. PIP 02368, PICT00-03-08580 and PICT0203-10718, and by a scientist exchange program between Germany and Argentina funded jointly by DAAD under grant No. DE/04/27956 and ANTORCHAS under grant No. 4248-6.
[1] Proceedings of the 17th International Conference on Ultra Relativistic Nucleus-Nucleus Collisions, edited by H.G. Ritter and X. N. Wang (IOP, Bristol, 2004); J. Phys. G 30, S633 ( 2004).

[2] F. Karsch, K. Redlich, and A. Tawfik, Eur. Phys. J. C 29, 549 (2003).

[3] E. Shuryak, J. Phys. G 30, S1221 (2004); Prog. Part. Nucl. Phys. 53, 273 (2004).

[4] D. B. Blaschke and K. A. Bugaev, Fiz. B 13, 491 (2004); Prog. Part. Nucl. Phys. 53, 197 (2004).

[5] K. Rajagopal and F. Wilczek, in At the Frontier of Particle Physics. Handbook of QCD, edited by M. Shifman (World Scientific, Singapore, 2001); M. Alford, Annu. Rev. Nucl. Part. Sci. 51, 131 (2001); D. Rischke, Prog. Part. Nucl. Phys. 52, 197 (2004).

[6] Physics of Neutron Star Interiors, edited by D. Blaschke, N. K. Glendenning, and A. Sedrakian, Lecture Notes in Physics Vol. 578 (Springer, New York, 2001); Superdense QCD Matter and Compact Stars, edited by D. Blaschke and D. Sedrakian, NATO Science Series II, Vol. 197 (Springer, New York, 2006).

[7] T. Klähn et al., nucl-th/0602038.

[8] D. Bailin and A. Love, Phys. Rep. 107, 325 (1984).

[9] M. Alford, K. Rajagopal, and F. Wilczek, Phys. Lett. B 422, 247 (1998); R. Rapp, T. Schäfer, E. V. Shuryak, and
M. Velkovsky, Phys. Rev. Lett. 81, 53 (1998).

[10] K. Iida and G. Baym, Phys. Rev. D 63, 074018 (2001); 66, 059903(E) (2002).

[11] M. Alford and K. Rajagopal, J. High Energy Phys. 06 (2002) 031.

[12] C. Gocke, D. Blaschke, A. Khalatyan, and H. Grigorian, hep-ph/0104183.

[13] S. B. Rüster, V. Werth, M. Buballa, I. A. Shovkovy, and D. H. Rischke, Phys. Rev. D 72, 034004 (2005).

[14] D. Blaschke, S. Fredriksson, H. Grigorian, A. M. Öztas, and F. Sandin, Phys. Rev. D 72, 065020 (2005).

[15] H. Abuki and T. Kunihiro, Nucl. Phys. A 768, 118 (2006).

[16] M. Baldo, M. Buballa, F. Burgio, F. Neumann, M. Oertel, and H. J. Schulze, Phys. Lett. B 562, 153 (2003).

[17] M. Buballa, Phys. Rep. 407, 205 (2005).

[18] I. Shovkovy and M. Huang, Phys. Lett. B 564, 205 (2003).

[19] D. Blaschke, S. Fredriksson, H. Grigorian, and A. M. Öztas, Nucl. Phys. A 736, 203 (2004).

[20] H. Grigorian, D. Blaschke, and D. N. Aguilera, Phys. Rev. C 69, 065802 (2004).

[21] D. N. Aguilera, D. Blaschke, and H. Grigorian, Nucl. Phys. A 757, 527 (2005).

[22] P. F. Bedaque, Nucl. Phys. A 697, 569 (2002).

[23] I. Shovkovy, M. Hanauske, and M. Huang, Phys. Rev. D 67, 103004 (2003). 
[24] F. Neumann, M. Buballa, and M. Oertel, Nucl. Phys. A 714, 481 (2003).

[25] T. Schäfer and E. V. Shuryak, Rev. Mod. Phys. 70, 323 (1998).

[26] C. D. Roberts and A. G. Williams, Prog. Part. Nucl. Phys. 33, 477 (1994); C. D. Roberts and S. M. Schmidt, Prog. Part. Nucl. Phys. 45, S1 (2000).

[27] J. Skullerud, D. B. Leinweber, and A. G. Williams, Phys. Rev. D 64, 074508 (2001).

[28] U. Vogl and W. Weise, Prog. Part. Nucl. Phys. 27, 195 (1991); S. Klevansky, Rev. Mod. Phys. 64, 649 (1992); T. Hatsuda and T. Kunihiro, Phys. Rep. 247, 221 (1994).

[29] E. Ruiz Arriola and L. L. Salcedo, Phys. Lett. B 450, 225 (1999).

[30] D. Blaschke, Y. L. Kalinovsky, G. Röpke, S. M. Schmidt, and M. K. Volkov, Phys. Rev. C 53, 2394 (1996).

[31] G. Ripka, Nucl. Phys. A 683, 463 (2001); R. S. Plant and M. C. Birse, Nucl. Phys. A 703, 717 (2002).

[32] H. Ito, W. Buck, and F. Gross, Phys. Rev. C 43, 2483 (1991); 45, 1918 (1992).

[33] M. Buballa and S. Krewald, Phys. Lett. B 294, 19 (1992).

[34] R. D. Bowler and M. C. Birse, Nucl. Phys. A 582, 655 (1995); R. S. Plant and M. C. Birse, Nucl. Phys. A 628, 607 (1998).

[35] A. Scarpettini, D. Gomez Dumm, and N. N. Scoccola, Phys. Rev. D 69, 114018 (2004).

[36] W. Broniowski, B. Golli, and G. Ripka, Nucl. Phys. A 703, 667 (2002).

[37] A. H. Rezaeian, N. R. Walet, and M. C. Birse, Phys. Rev. C 70, 065203 (2004).

[38] A.H. Rezaeian and H.-J. Pirner, Nucl. Phys. A 769, 35 (2006).

[39] D. Blaschke and P.C. Tandy, in Understanding Deconfinement in QCD (World Scientific, Singapore, 2000), p. 218.
[40] I. General, D. Gómez Dumm, and N. N. Scoccola, Phys. Lett. B 506, 267 (2001); D. Gómez Dumm and N. N. Scoccola, Phys. Rev. D 65, 074021 (2002).

[41] D. Gómez Dumm and N. N. Scoccola, Phys. Rev. C 72, 014909 (2005).

[42] R. S. Duhau, A. G. Grunfeld, and N. N. Scoccola, Phys. Rev. D 70, 074026 (2004).

[43] D. Blaschke, H. Grigorian, A. Khalatyan, and D. N. Voskresensky, Nucl. Phys. B, Proc. Suppl. 141, 137 (2005).

[44] G. Ripka, Quarks Bound by Chiral Fields (Oxford University Press, Oxford, 1997).

[45] S. M. Schmidt, D. Blaschke, and Y.L. Kalinovsky, Phys. Rev. C 50, 435 (1994).

[46] P. Amore, M. C. Birse, J. A. McGovern, and N. R. Walet, Phys. Rev. D 65, 074005 (2002).

[47] M. Buballa and I. A. Shovkovy, Phys. Rev. D 72, 097501 (2005).

[48] D. Gómez Dumm, A. G. Grunfeld, and N. N. Scoccola (unpublished).

[49] R. T. Cahill, Nucl. Phys. A 543, 63C (1992), and Refs. therein.

[50] R. Alkofer, A. Holl, M. Kloker, A. Krassnigg, and C.D. Roberts, Few Body Syst. 37, 1 (2005).

[51] N. K. Glendenning, Phys. Rev. D 46, 1274 (1992).

[52] T. Tatsumi, M. Yasuhira, and D. N. Voskresensky, Nucl. Phys. A 718, 359 (2003).

[53] S. Reddy and G. Rupak, Phys. Rev. C 71, 025201 (2005).

[54] A. Gerhold and A. Rebhan, Phys. Rev. D 68, 011502 (2003).

[55] A. Kryjevski, Phys. Rev. D 68, 074008 (2003).

[56] D. D. Dietrich and D. H. Rischke, Prog. Part. Nucl. Phys. 53, 305 (2004).

[57] D. Blaschke, C. D. Roberts, and S. M. Schmidt, Phys. Lett. B 425, 232 (1998). 